\title{
Proyecto LIFE SAMFIX:
}

Primeros resultados de la comunidad de insectos forestales capturada en trampeos para detectar la especie invasora Xylosandrus crassiusculus (Coleoptera, Curculionidae, Scolytinae)

\section{LIFE SAMFIX projet:}

First results of the forest insect community captured in traps to detect the invasive species Xylosandrus crassiusculus (Coleoptera, Curculionidae, Scolytinae)

Gallego, D. ${ }^{1 *}$; Molina, N. ${ }^{1}$; Di Sora, N. ${ }^{1}$; Mas, H. ${ }^{2}$; González, E. ${ }^{3}$

${ }^{1}$ Departamento de Ecología, Universidad de Alicante, Sant Vicent del Raspeig, 03690, Alicante ${ }^{2}$ CIEF VAERSA, Avda Comarques del País Valencià, 114, 46930, Quart de Poblet, València. ${ }^{3}$ SILCO S.L., c/ Escalinata, 28440, Guadarrama, Madrid 


\title{
Resumen
}

Xylosandrus crassiusculus es una especie exótica de escolítido de ambrosía que se detectó en la península ibérica por primera vez en 2016 en unos algarrobos atacados en un área residencial, en Benifaió, a unos 20 km de la ciudad de Valencia, colindante con el Parque Natural Municipal El Tello (Llombai). Tan sólo se han registrado ataques de X. crassiusculus sobre algarrobo, causando el secado de ramillos, la muerte de ramas e incluso la de la totalidad de parte aérea del árbol, aunque sin mermar la capacidad de rebrote. Se trata de un insecto muy polífago, con hospedadores como Diospyros kaki Thunb, Ficus carica L., Malus domestica (Borkh.) Borkh., Prunus avium (L.) L., Prunus domestica L., Prunus persica (L.) Batsch, y los géneros Alnus, Quercus, Populus, Salix, Ulmus, entre otros. El proyecto SAMFIX tiene el objetivo desarrollar de forma colaborativa competencias e investigación para implementar protocolos y herramientas de gestión de dos especies exóticas: $X$. crassiusculus y X. compactus. Cuenta con socios de Italia, Francia y España. La Universidad de Alicante es el socio español, Departamento de Ecología y el Instituto Universitario de investigación CBIO, colaborando con el Servicio de Ordenación y Gestión Forestal de la Generalitat Valenciana. Se presentan los primeros resultados relativos a la comunidad de saproxílicos asociada a X. craussiusculus, enmarcada en las tareas C3 y D2 del proyecto SAMFIX. Hasta la fecha se ha caracterizado una comunidad compleja, compuesta por una horquilla de 40 a 50 especies, que incluye xilófagos, floemófagos, escolítidos de ambrosía autóctonos, depredadores, depredadores facultativos, e incluso otras especies invasoras ya establecidas como Xyleborus ferrugineus.

Palabras clave: escolítidos de ambrosía, insectos saproxilófagos, Ceratonia siliqua L., Crosstrap, alfa-pineno, etanol.

\begin{abstract}
Xylosandrus crassiusculus is an alien ambrosia beetle species detected for the first time in Iberian Peninsula in 2016, attacking a few carob trees in a residential area of Benifaió, near of Valencia, surrounding of the Parque Natural Municipal de El Tello (Llombai). Only attacks on carob trees have been observed, causing death of fine and big branches, and in only a case the entire tree death, although without diminishing their regrowing ability. They are insects with a wide host spectrum: Diospyros kaki Thunb, Ficus carica L., Malus domestica (Borkh.) Borkh., Prunus avium (L.) L., Prunus domestica L., Prunus persica (L.) Batsch, and the genus Alnus, Quercus, Populus, Salix, Ulmus, among others. The LIFE SAMFIX project is aimed to developing collaborative competences and research to develop management protocols and tools for the alien species $X$. crassiusculus and $X$. compactus. The project has partners in Italy, France and Spain. The Spanish partner is the University of Alicante (Department of Ecology and CBIO Institute), in collaboration with the Forest Planning and Management Service of the Valencian Regional Government. First results of the saproxylic beetle community analyses associated to $X$. crassiusculus are shown. A highly complex community emerged from our results, composed so far by 40 to 50 species, including xylophagous, floemophagous, native ambrosia beetles, predators, intraguild predators, and other alien species such as Xyleborus ferrugineus.
\end{abstract}

Keywords: Ambrosia beetles, saproxylic insects, Ceratonia siliqua, Crosstrap, alfa-pinene, ethanol. 


\section{Introducción}

Los escolítidos de ambrosía (Coleoptera, Curculionidae, Scolytinae) se encuentran entre los principales grupos de insectos con capacidad de generar invasiones biológicas que podrían causar pérdidas económicas significativas así como importantes desequilibrios en los ecosistemas de todo el mundo (Brockerhoff y Leibhold, 2017). Estos insectos tienen como hábito de vida la xilomicetofagia, excavando galerías profundas en vegetales leñosos vivos donde encuentran las condiciones idóneas de humedad y temperatura para cultivar hongos que ellos mismos transportan, los hongos de ambrosía, en órganos especializados llamados micangios. Los escarabajos crían su descendencia en estas galerías, alimentándose del hongo. Cuando emergen los nuevos adultos, sus micangios estarán cargados de esporas de hongo dispuestos a colonizar nuevos vegetales. Debido a esta forma de vida estos escarabajos son fácilmente transportados por medio del comercio mundial en muchos tipos de material vegetal, como planta viva destinada a jardinería o agricultura, madera en rollo, madera de embalaje o estiba. Permanecen ocultos dentro de la madera o el material vegetal y pueden escapar fácilmente a los controles fitosanitarios, así como sobrevivir a condiciones climáticas adversas durante los viajes por mar (Rassati et al., 2015). Además el grupo de los Xyleborini se reproducen mediante una combinación de haplodiploidía y endogamia. Por lo general, los machos son haploides procedentes de huevos no fertilizados y permanecen toda su vida en el interior de galerías, mientras que las hembras son diploides y provienen de huevos fertilizados y tienen capacidad de vuelo y por tanto de dispersarse. Esto permite que una única hembra sea capaz de establecer poblaciones exitosas en lugares no nativos, lo que les dota de una enorme capacidad invasiva (Kirkendall, 1983).

En septiembre de 2016, en el Parque Nacional del Circeo (Italia), incluido en la Red Natura 2000, se descubrió por primera vez en un espacio natural europeo una extensa infestación de dos especies invasoras de escolítidos de ambrosía asiáticos: Xylosandrus compactus (Eichhoff 1876) y X. craussiusculus (Coleoptera, Curculionidae, Scolytinae) (Vannini et al., 2017). Las plantas atacadas mostraron síntomas como marchitamiento, muerte de ramillos, rotura de ramas y decaimiento generalizado. Como estado final, la maquia mediterránea atacada puede mostrar un marchitamiento difuso, que es lo que se está observando en un área de unas 8 ha en el Parque Nacional del Circeo.

Xylosandrus compactus fue encontrado en 2011 en las regiones italianas de Campania, Toscana y Liguria (Nageleisen et al., 2014). Se encontró en Francia en Saint Tropez y Saint-Jean-Cap-Ferrat en 2016 y en 2017 en el Jardín Botánico de Villa Thuret in Antibes (Chapin et al., 2016). Por otro lado X. crassiusculus se encontró por primera vez en Europa en el norte de Italia (Pennachio et al., 2003), en 2014 en Mont Boron y en 2016 en la Reserva Biológica de Sainte Marguerite (Francia).

En 2016, un ciudadano alertó sobre la presencia de cuatro algarrobos aparentemente muertos en la urbanización El Pla de les Cotxes, Benifaió (Valencia), en una zona de rambla que funciona como zona verde. Esta zona proviene de un antiguo 
cultivo abandonado de algarrobos que en la actualidad está cubierta por una formación mixta de pinar de pino carrasco y garriga mediterránea con lentisco, aladierno, y otros matorrales nobles. Los árboles más afectados estaban completamente cubiertos de pequeños agujeros, mientras que otros presentaban exudados o cilindros compactos de serrín blanco que emergían de ellos. Se capturaron insectos que fueron identificados como $X$. craussiusculus (Gallego et al., 2017).

En Valencia tan sólo se han registrado ataques de $X$. craussiusculus sobre algarrobo, causando el secado de ramillos, la muerte de ramas e incluso la de la totalidad de la parte aérea del árbol, aunque sin mermar la capacidad de rebrote. No obstante se trata de un insecto muy polífago, y en la zona se encuentran como hospedadores potenciales a especies como D. kaki, F. carica, M. domestica, P. avium, P. domestica, P. persica, y los géneros Alnus, Quercus, Populus, Salix, Ulmus. Todas estas plantas potencialmente hospedadoras son de interés agrícola o forestal.

SAMFIX (Saving Mediterranean Forests from Invasions of Xylosandrus beetles and associated pathogenic fungi) es un proyecto europeo cofinanciado por el programa LIFE17 NAT/IT/000609, que comenzó en julio de 2018 y finalizará en febrero de 2022. SAMFIX pretende desarrollar protocolos específicos y herramientas para la prevención y alerta temprana de invasiones de especies del género Xylosandrus. Este proyecto está liderado por el Parque Nacional del Circeo (Italia) y cuenta como socios a varias organizaciones italianas, tanto públicas como privadas, organizaciones públicas francesas y a la Universidad de Alicante como único socio español. Desde la Universidad de Alicante participa el Departamento de Ecología (coordinador) y el Instituto Universitario de investigación CBIO. Como entidad colaboradora participa el Servicio de Ordenación y Gestión forestal (Direcció General de Medi Natural i d'Avaluació Ambiental, Consellera d'Agricultura, Medi Ambient, Canvi Climàtic i Desenvolupament Rural).

Las Acciones C y D del proyecto SAMFIX pretenden establecer protocolos para la detección temprana de eventos de invasión y contención de las poblaciones establecidas, así como también evaluar el impacto en las comunidades de insectos en los que estas especies se integran e interactúan. El presente trabajo presenta los resultados parciales de la primera campaña de estas acciones C y D.

\section{Material y Métodos}

Se instalaron 40 trampas Crosstrap ${ }^{\circledR}$ (Econex, Murcia), en la zona de estudio del proyecto (Fig. 1), cebadas con alfa-pineno y etanol, de acuerdo con Miller y Rabaglia (2009). Las trampas fueron revisadas cada 15 días, entre marzo y septiembre de 2019. Los cebos se repusieron de acuerdo con las indicaciones del fabricante. Se utilizaron capturas en seco mediante tarro de capturas con fondo de malla. En cada tarro se introdujo un fragmento de malla impregnada en deltametrina para impedir al máximo el escape de las capturas, y reducir los daños por depredación entre los insectos capturados. 


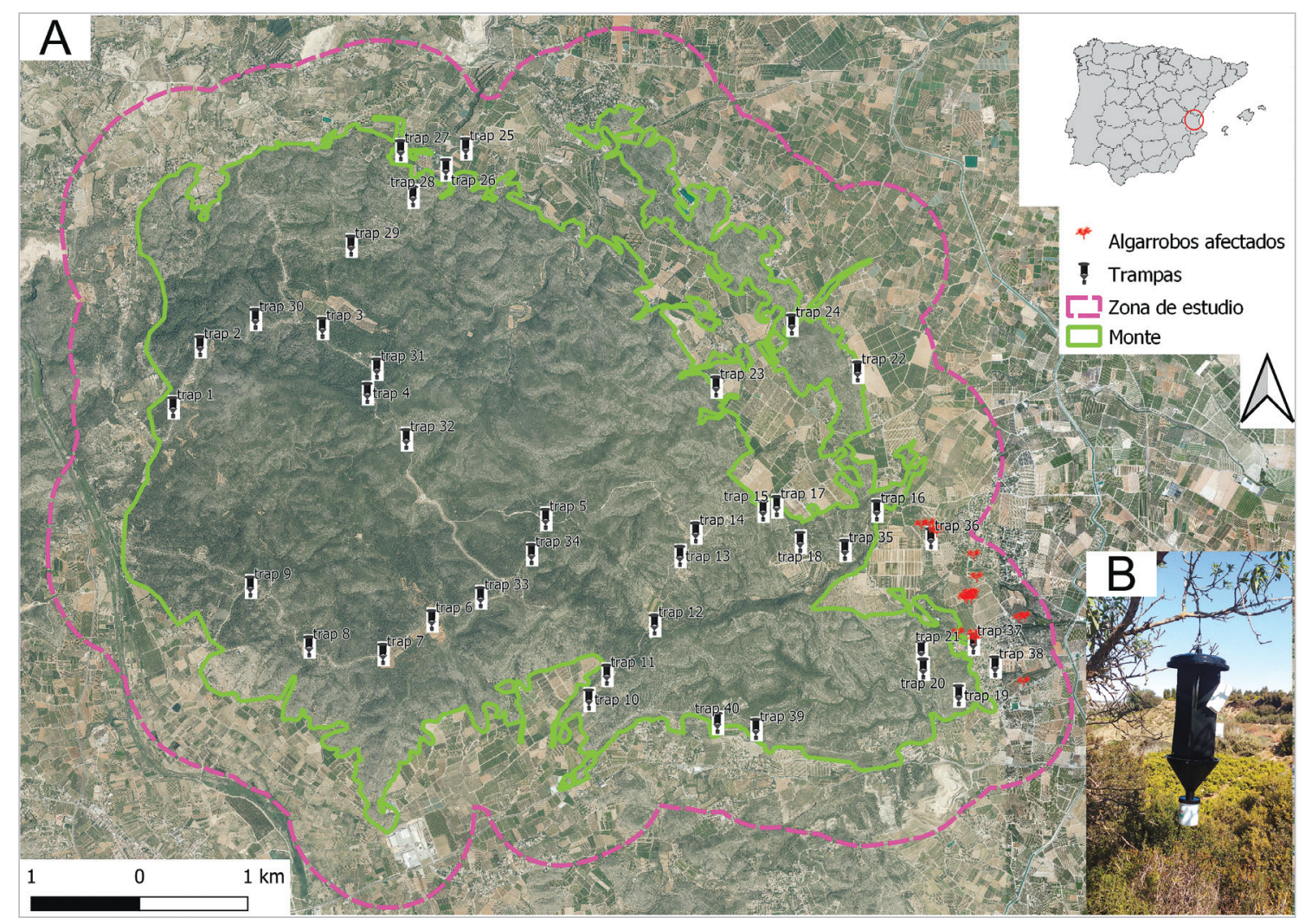

Figura 1. A: Ubicación de las trampas en la zona de estudio del proyecto SAMFIX. B: y Modelo de trampa Crosstrap (Econex, Murcia) usada.

Las muestras de campo fueron conservadas en etanol al 70\% y transportadas a laboratorio, donde se realizó el recuento de todos los individuos bajo lupa binocular con zoom de hasta 80 aumentos, identificando su especie mediante una colección de comparación que se encuentra depositada en el laboratorio del Departamento de Ecología de la Universidad de Alicante. Los diferentes gremios tróficos se han establecido en función de la información disponible en Gallego y Campo (2010) y Más (2015).

\section{Resultados}

El recuento total de ejemplares capturados fue de 10816 individuos distribuyéndose en 88 especies repartidas en 23 familias. Además de $X$. crassiusculus se han capturado otras 6 especies exóticas.

La Tabla 1 muestra el número de especies por cada grupo taxonómico considerado. El grupo más representado es Scolytinae, con 23 especies, seguido por Cerambycidae con 13 especies y Bostrichidae con 11 especies. El resto de las 20 familias están poco representadas, con cuatro o menos especies por grupo. 
Tabla 1. Número de especies por grupo taxonómico capturadas durante el trampeo, en la zona de estudio del proyecto (Fig. 1), entre marzo y septiembre de 2019.

\begin{tabular}{cc|cc}
\hline TAXÓN & NÚMERO DE ESPECIES & TAXÓN & NÚMERO DE ESPECIES \\
\hline Scolytinae & 23 & Lyctidae & 2 \\
\hline Cerambycidae & 13 & Anamorphidae & 1 \\
\hline Bostrichidae & 11 & Anthribidae & 1 \\
Coccinellidae & 4 & Bothrideridae & 1 \\
Tenebrionidae & 4 & Cossoninae & 1 \\
\hline Nitidulidae & 3 & Curculioninae & 1 \\
\hline Cleridae & 3 & Dryophtoridae & 1 \\
Buprestidae & 3 & Elateridae & 1 \\
Cetoniidae & 3 & Histeridae & 1 \\
\hline Carabidae & 2 & Monotomidae & 1 \\
\hline Mycetophagidae & 2 & Zopheridae & 1 \\
\hline Ptinidae & 2 & & \\
\hline
\end{tabular}

La Tabla 2 presenta una lista detallada de todas las especies encontradas incluyendo el vegetal hospedador sobre el que se reproduce y el gremio trófico al que pertenece la especie. La mayor parte de las especies son muy polífagas, con muy poca especificidad de hospedador. También destaca el grupo de especies cuyo hospedador son las coníferas, particularmente cualquier especie de Pinus, representada en la zona de estudio por Pinus halepensis Mill. Tan sólo cuatro especies de escolítidos pueden considerase oligófagas: Hypoborus ficus atacando a Ficus carica, Chaetoptelius vestitus sobre Pistacea lentiscus L., Phloeotribus scarabaeoides sobre Olea europea L. y Xylocleptes bispinus sobre Clematis spp.

Entre los grupos tróficos destacan las especies de hábitos xilófagos y floemófagos, seguidos por depredadores y depredadores facultativos. Unas pocas especies son de hábitos detritivoros, se capturaron además, cuatro especies xilomicetófagas y una especie carpófaga.

Destaca la captura de especies del género Cerambyx, sobre todo teniendo en cuenta que en la zona de estudio tan sólo está presente Quercus coccifera L., especie no colonizada por estos cerambícidos.

\section{4.- Discusión}

La comunidad de saproxilófagos, constituida por 88 especies, resulta altamente compleja si se compara con los resultados obtenidos en otros datos del levante peninsular, como por ejemplo las 23 familias y 71 especies registradas en el Parque Regional de Sierra Espuña, en Murcia, en trabajos realizados en una superficie de territorio similar y con técnicas similares (Gallego, 2019). Los datos que se muestran en este trabajo apenas cubren 6 meses de trampeo, mientras que los de Sierra Espuña se mantuvieron durante 40 meses. Tan alta diversidad recogida en un espa- 
Tabla 2. Lista de especies por taxón considerado. En sombreado gris y negrita se resaltan las especies exóticas.

\begin{tabular}{|c|c|c|c|}
\hline TAXÓN & ESPECIES & HOSPEDADOR & GREMIO \\
\hline Anamorphidae & Symbiotes gibberosus (Lucas, 1849) & Varios & Detritívoro \\
\hline Anthribidae & Araecerus coffeae (Fabricius, 1801) & Varios & Detritívoro \\
\hline \multirow[t]{11}{*}{ Bostrichidae } & Apate monachus Fabricius, 1775 & Varios & Xilófago \\
\hline & Xylopertha praeusta (Germar, 1817) & Varios & Xilófago \\
\hline & Xylopertha retusa (Olivier, 1790) & Varios & Xilófago \\
\hline & Scobicia pustulata (Fabricius, 1801) & Varios & Xilófago \\
\hline & Scobicia chevrieri (Villa \& Villa, 1835) & Varios & Xilófago \\
\hline & Sinoxylon sexdentatum (Olivier, 1790) & Varios & Xilófago \\
\hline & Xyloperthella picea (Olivier, 1790) & Varios & Xilófago \\
\hline & Xyloperthella crinitarsis (Imhoff, 1843) & Varios & Xilófago \\
\hline & Stephanopachys quadricollis (Marseul, 1879) & Varios & Xilófago \\
\hline & Rhyzopertha dominica (Fabricius, 1792) & Varios & Detritívoro \\
\hline & Trogoxylon impressum (Comolli 1837) & Varios & Xilófago \\
\hline Bothrideridae & $\begin{array}{l}\text { Ogmoderes angusticollis } \\
\text { (Brisout de Barneville, 1861) }\end{array}$ & Varios & Depredador \\
\hline \multirow[t]{4}{*}{ Buprestidae } & Chrysobothris solieri Laporte \& Gory, 1839 & Pinus spp & $\begin{array}{l}\text { Depredador } \\
\text { facultativo }\end{array}$ \\
\hline & Phaenops cyaena (Fabricius, 1775) & Pinus spp & $\begin{array}{l}\text { Depredador } \\
\text { facultativo }\end{array}$ \\
\hline & Buprestis octoguttata Linnaeus, 1758 & Pinus spp & $\begin{array}{l}\text { Depredador } \\
\text { facultativo }\end{array}$ \\
\hline & Acmaeodera bipunctata (Olivier, 1790) & Varios & $\begin{array}{l}\text { Depredador } \\
\text { facultativo }\end{array}$ \\
\hline \multirow[t]{2}{*}{ Carabidae } & Dromius chobauti Puel 1923 & Varios & Depredador \\
\hline & $\begin{array}{l}\text { Metadromius ramburii } \\
\text { (Piochard de la Brûlerie 1867) }\end{array}$ & Varios & Depredador \\
\hline \multirow[t]{11}{*}{ Cerambycidae } & Arhopalus ferus (Mulsant, 1839) & Pinus spp & $\begin{array}{l}\text { Depredador } \\
\text { facultativo }\end{array}$ \\
\hline & Arhopalus rusticus (Linnaeus, 1758) & Pinus spp & $\begin{array}{l}\text { Depredador } \\
\text { facultativo }\end{array}$ \\
\hline & Arhopalus syriacus (Reitter, 1895) & Pinus spp & $\begin{array}{l}\text { Depredador } \\
\text { facultativo }\end{array}$ \\
\hline & Monochamus galloprovincialis (Olivier, 1795) & Pinus spp & $\begin{array}{l}\text { Depredador } \\
\text { facultativo }\end{array}$ \\
\hline & Cerambyx welensii (Kuster, 1846) & Varios & Xilófago \\
\hline & Cerambyx miles Bonelli, 1812 & Pinus spp & Xilófago \\
\hline & Oxypleurus nodieri Mulsant, 1839 & Pinus spp & Xilófago \\
\hline & Clytus arietis (Linnaeus, 1758) & Varios & Xilófago \\
\hline & Stromatium unicolor (Olivier 1795) & Varios & Xilófago \\
\hline & Trichoferus fasciculatus (Faldermann 1837) & Varios & Xilófago \\
\hline & Nathrius brevipennis (Mulsant 1839) & Varios & Xilófago \\
\hline
\end{tabular}


Viene de pág. anterior

\begin{tabular}{|c|c|c|c|}
\hline TAXÓN & ESPECIES & HOSPEDADOR & GREMIO \\
\hline & Penichroa timida Menetriés, 1832 & Varios & Xilófago \\
\hline & Chlorophorus pilosus (Forster, 1771) & Varios & Xilófago \\
\hline & Xylotrechus stebbingi Gahan, 1906 & Varios & Xilófago \\
\hline \multirow[t]{3}{*}{ Cetoniidae } & Protaetia cuprea (Fabricius 1775) & Varios & Detritívoro \\
\hline & Protaetia oblonga (Gory \& Percheron, 1833) & Varios & Detritívoro \\
\hline & Protaetia morio (Fabricius, 1781) & Varios & Detritívoro \\
\hline \multirow[t]{3}{*}{ Cleridae } & Opilo abeillei Korge 1960 & Pinus spp & Depredador \\
\hline & Thanasimus formicarius (Linnaeus, 1758) & Pinus spp & Depredador \\
\hline & Denops albofasciatus (Charpentier, 1825) & Varios & Depredador \\
\hline \multirow[t]{4}{*}{ Coccinellidae } & Nephus quadrimaculatus (Herbst 1783) & Varios & Depredador \\
\hline & Chilocorus bipustulatus (Linnaeus 1758) & Varios & Depredador \\
\hline & Rhyzobius lophanthae (Blaisdell 1892) & Varios & Depredador \\
\hline & Rhyzobius chrysomeloides (Herbst 1792) & Varios & Depredador \\
\hline Cossoninae & Rhyncolus sp & Pinus spp & Floemófago \\
\hline Curculioninae & Cionus longicollis C. Brisout, 1863 & Varios & Ramoneador \\
\hline Dryophtoridae & Rhynchophorus ferrugineus (Olivier, 1790) & Palmaceas & Perforador \\
\hline Elateridae & Lacon punctatus (Herbst, 1779) & Pinus spp & $\begin{array}{l}\text { Depredador } \\
\text { facultativo }\end{array}$ \\
\hline Histeridae & Teretrius parasita Marseul 1863 & Varios & Depredador \\
\hline \multirow[t]{2}{*}{ Lyctidae } & Lyctus brunneus (Stephens, 1830) & Varios & Xilófago \\
\hline & Lyctus linearis (Goeze, 1777) & Varios & Xilófago \\
\hline Monotomidae & Rhyzophagus ferrugineus (Paykull, 1800) & Pinus spp & Depredador \\
\hline \multirow[t]{2}{*}{ Mycetophagidae } & Litargus connexus (Geoffroy 1785) & Varios & Detritívoro \\
\hline & Litargus balteatus Leconte 1856 & Varios & Detritívoro \\
\hline \multirow[t]{3}{*}{ Nitidulidae } & Pityophaugus laevior Abeille, 1872 & Pinus spp & Depredador \\
\hline & Phenolia picta (MacLeay 1825) & Varios & Detritívoro \\
\hline & Haptoncus luteolus (Erichson, 1843) & Varios & Frugívoro \\
\hline \multirow[t]{2}{*}{ Ptinidae } & Stegobium paniceum (Linnaeus, 1758) & Varios & Detritívoro \\
\hline & Ozognathus cornutus (Le Conte 1859) & Varios & Detritívoro \\
\hline \multirow[t]{10}{*}{ Scolytinae } & $\begin{array}{l}\text { Xylosandrus crassiusculus } \\
\text { (Motschulsky, 1866) }\end{array}$ & Varios & Xilomicetófago \\
\hline & Orthotomicus erosus (Wollaston, 1857) & Pinus spp & Floemófago \\
\hline & Xyleborinus saxesenii (Ratzeburg, 1837) & Varios & Xilomicetófago \\
\hline & Hylurgus miklitzi Wachtl, 1881 & Pinus spp & Floemófago \\
\hline & Hylurgus ligniperda (Fabricius, 1787) & Pinus spp & Floemófago \\
\hline & Hypoborus ficus Erichson, 1836 & Ficus carica & Floemófago \\
\hline & Crypturgus numidicus Ferrari, 1867 & Pinus spp & Floemófago \\
\hline & Crypturgus mediterraneus Eichhoff, 1871 & Pinus spp & Floemófago \\
\hline & Carphoborus pini Eichhoff, 1881 & Pinus spp & Floemófago \\
\hline & $\begin{array}{l}\text { Dactylotrypes longicollis } \\
\text { (Wollaston, 1864) }\end{array}$ & Palmaceas & Carpófago \\
\hline
\end{tabular}


Viene de pág. anterior

\begin{tabular}{|c|c|c|c|}
\hline TAXÓN & ESPECIES & HOSPEDADOR & GREMIO \\
\hline & Chaetoptelius vestitus (Mulsant \& Rey, 1860) & $\begin{array}{l}\text { Pistacia } \\
\text { lentiscus }\end{array}$ & Floemófago \\
\hline & Pityogenes calcaratus (Eichhoff, 1878) & Pinus spp & Floemófago \\
\hline & Xylocleptes bispinus (Duftschmid, 1825) & Clematis spp. & Floemófago \\
\hline & Liparthrum genistae (Aube, 1862) & Varios & Floemófago \\
\hline & Xyleborini sp & Varios & Xilomicetófag \\
\hline & Phloeotribus scarabaeoides (Bernard, 1788) & Olea europaea & Floemófago \\
\hline & Tomicus destruens (Wollaston, 1865) & Pinus spp & Floemófago \\
\hline & Hylastes linearis Erichson, 1836 & Pinus spp & Floemófago \\
\hline & Pteleobius kraatzi (Eichhoff, 1864) & Varios & Floemófago \\
\hline & Xyleborus ferrugineus (Fabricius, 1801) & Varios & Xilomicetófag \\
\hline & Pityophthorus pubescens (Marsham, 1802) & Varios & Floemófago \\
\hline & Pityophthorus sp & Varios & Floemófago \\
\hline & Scolytus amygdali Guerin, 1847 & Prunus spp & Floemófago \\
\hline \multirow[t]{4}{*}{ Tenebrionidae } & Corticeus pini (Panzer, 1799) & Pinus spp & Depredador \\
\hline & Lagria grenieri Brisout, 1868 & Varios & Ramoneador \\
\hline & Pentaphyllus testaceus (Hellwig 1792) & Varios & Detritívoro \\
\hline & Nesotes carbo (Küster 1850) & Varios & Detritívoro \\
\hline Zopheridae & Aulonium ruficorne (Olivier, 1790) & Pinus spp & Depredador \\
\hline
\end{tabular}

cio de tiempo tan corto es indicadora de la enorme variabilidad ambiental de la zona de estudio. Sierra Espuña, aunque acoja también una gran variedad de ambientes, está cubierta casi en su totalidad de bosques de coníferas. En cambio, en la zona estudio existen formaciones de pinar y formaciones de garriga con una importante presencia de algarrobos procedentes de antiguos cultivos abandonados en la década de 1970. Teniendo en cuenta que por el momento sólo se dispone de resultados preliminares, la presencia de ejemplares maduros de frondosas puede ser la razón por la que se haya detectado un número tan alto de especies. De acuerdo con esta hipótesis, serían los algarrobos los que permitirían la presencia de las especies de Cerambyx en ambientes sin Quercus hospedadores adecuados. También se entendería la presencia del depredador Ogmoderes angusticollis, una especie incluida en la lista roja de UICN (Brustel et al., 2017), con la categoría de preocupante. Esta especie está considerada como un relicto de la fauna preglacial que habita en bosques templados sobre $C$. siliqua, F. carica, P. lentiscus y el género Quercus, con hábitos depredadores sobre bostríquidos. Esta cita de la especie O. angusticollis amplía mucho su distribución, ya que en la península ibérica tan sólo se conocía en Cataluña, en las provincias de Barcelona y Gerona.

Es muy destacable la detección de seis especies exóticas además de $X$. craussiusculus, la especie que ha motivado el estudio. En la familia Bostrichidae, se han encontrado Apate monachus y Xyloperthella crinitarsis, dos especies de origen afri- 
cano subtropical muy polífagas. Se han observado numerosos ataques de $A$. monachus en algarrobo, y son conocidas las pérdidas económicas que causa esta especie en plantaciones de Punica granatum. Se han capturado muchos menos ejemplares de $X$. crinitarsis, desconociendose el hospedador u hospedadores en la zona de estudio. El cerambícido exótico Xylotrechus stebbigi es una especie originaria de Asia, de la zona sur del Himalaya, es muy polífaga y se desconoce qué planta utiliza como hospedadora. Se han capturado, así mismo, individuos de especies exóticas que ya llevan tiempo instaladas en la península ibérica como Rhynchophorus ferrugineus y los nitidúlidos Phenolia picta y Hapcontus luteolus. Los tremendos cambios sobre la distribución de las palmeras causada por la primera especie son altamente conocidos, mientras que las otras dos causan daños en frutas.

Entre los escolítidos, son dos especies de ambrosía exóticas las que se han capturado en la zona de estudio: X. crassiusculus y Xyleborus ferrugineus. Esta última especie, de origen norteamericano, está incluida en la lista A2 de la Asia and Pacific Plant Protection Commission (APPPC), donde ataca a cacao y café; fue citada por primera vez para Europa en 2011, tras haber sido capturado en 2009 en las proximidades de la ciudad de Murcia, en trampas cebadas con $\alpha$-pineno y etanol (Gallego et al., 2011). Se desconoce la planta hospedadora que acoge este insecto en la zona de estudio. A lo largo del proyecto, que finalizará en 2022, se profundizará en el conocimiento de los roles ecológicos de las especies, su interacción con el resto de las especies, así como el impacto en la estructura y funciones ecosistémicas de $X$. crassiusculus para desarrollar protocolos necesarios para prevenir y contener futuras invasiones de estos insectos.

\section{Conclusiones}

La comunidad de saproxilófagos en la que está integrado $X$. crassiusiculus es compleja, compuesta principalmente por xilófagos, floemófagos, depredadores y depredadores facultativos con mayor número de especies en los taxones Scolytinae, Cerambycidae y Bostrichidae y con la presencia destacable de especies exóticas.

\section{Agradecimientos}

Este trabajo ha sido cofinanciado por el programa LIFE de la Unión Europea en virtud del acuerdo de subvención LIFE17 NAT/IT/000609. Especial agradecimiento para José Luís Lencina por su asistencia en la determinación de muchas de las especies de este trabajo.

\section{Bibliografía}

Brockerhoff, E.G.; \& A.M., Liebhold.; 2017. Ecology of forest insect invasions. Biol. Invasions. 19, 3141-3159. https://doi.org/10.1007/s10530-017-1514-1 
Brustel, H.; Otero, J.; Audisio, P.; \& Micó Balaguer, E.; 2017. Ogmoderes angusticollis. The IUCN Red List of Threatened Species 2017.: e.T47173218A47696228. Downloaded on 25 November 2019. http://dx.doi.org/10.2305/IUCN.UK.2017-2.RLTS.T47173218A476 96228.en

Chapin, E.; Mouttet, R.; \& Chauvel, G.; 2016. "Xylosandrus compactus trouvé en France métropolitaine." Phytoma, 697, 10-12.

Gallego, D.; 2019. La entomofauna forestal de Espuña, un gran reservorio de biodivesidad. In: Giménez, L.; Muñoz, A.; Águila, M.; Yelo, N.; Velamazán, M.; Sánchez-Zapata, J.A.; (eds.) Sierra Espuña, naturaleza y cultura. Comunidad Autónoma de la Región de Murcia, Murcia, $800 \mathrm{pp}$.

Gallego, D.; Campo, M.T.; 2010. El bosque ante el cambio climático. Murcia Enclave Ambiental. 25, 16-27.

Gallego, D.; Lencina, J.L.; Mas, H.; Ceveró, J.; \& Faccoli, M.; 2017. First record of the granulate ambrosia beetle, Xylosandrus crassiusculus (Coleoptera: Curculionidae, Scolytinae), in the Iberian Peninsula. Zootaxa, 4273(3), 431-434. https://doi.org/10.11646/zootaxa. 4273.3.7

Gallego, D.; Knizek, M.; Campo, M.T.; 2011. Nuevas citas de especies de fauna exótica de interés forestal. En Actas II Reunión Científica del Grupo de Trabajo de Sanidad Forestal de la SECF. Plasencia.

Kirkendall, L.R.; 1983 The evolution of mating systems in bark and ambrosia beetles (Coleoptera: Scolytidae and Platypodidae). Zool J Linnean Soc. 77, 293-352. https://doi.org/ 10.1111/j.1096-3642.1983.tb00858.x

Mas, H.; 2015. Fenología y capacidad de dispersión de Monochamus galloprovincialis (Olivier 1795) en la Península Ibérica. Tesis doctoral. Universidad Politécnica de Madrid. 256 pp

Miller, D.R.; Rabaglia R.J.; 2009 Ethanol and (-)-alpha-Pinene: attractant kairomones for bark and ambrosia beetles in the southeastern US. J Chem Ecol. 35: 435-48. https://doi.org/ 10.1007/s10886-009-9613-9

Nageleisen, L.M.; Bouge, C.; \& Noblecourt, T.; 2014. Les Scolytes du genre Xylosandrus en France (Coleoptera, Curculionidae, Scolytinae). L'Entomologiste, 4, 267-271.

Pennachio, F.; Roversi, P.; Francardi, V;. \& Gatti, E.; 2003. Xylosandrus crassiusculus (Motschulsky), a bark beetle knew to Europe. Redia, 86, 77-80.

Rassati, D.; Faccoli, M.; Petrucco Toffolo, E.; Battisti, A.; Marini, L.; 2015. Improving the early detection of alien wood-boring beetles in ports and surrounding forests. J. Appl. Ecol. 52, 50-58. https://doi.org/10.1111/1365-2664.12347

Vannini, A.; Contarini, M.; Faccoli, M.; Valle, M. D.; Rodriguez, C. M.; Mazzetto, T.; et al., 2017. First report of the ambrosia beetle Xylosandrus compactus and associated fungi in the Mediterranean maquis in Italy, and new host-pest associations. EPPO Bulletin 47, 100-103. https://doi.org/10.1111/epp.12358 
